\title{
A short note on sign changes and non-vanishing of Fourier coefficients of half-integral weight cusp forms
}

\author{
Winfried Kohnen ${ }^{1}$
}

Received: 14 June 2021 / Published online: 14 December 2021

(c) The Author(s) 2021

\begin{abstract}
We study sign changes and non-vanishing of a certain double sequence of Fourier coefficients of cusp forms of half-integral weight.
\end{abstract}

Keywords Modular form $\cdot$ Half-integral weight $\cdot$ Sign changes

Mathematics Subject Classification $11 \mathrm{~F} 30 \cdot 11 \mathrm{~F} 37$

\section{Introduction}

Starting with the paper [6] many authors have investigated sign change properties of Fourier coefficients of cusp forms, in various directions. In particular, the case of half-integral weight has been the focus of much research. If $g$ is a cusp form of half-integral weight $k+\frac{1}{2}$ with real Fourier coefficients $c(m)(m \geq 1)$ and in addition $g$ is a Hecke eigenform, then there are at least two important themes in this area: on the one hand the study of sign changes of $\left(c\left(t n^{2}\right)\right)_{n \geq 1}$ where $t$ is a fixed positive integer, and on the other hand the corresponding question for the sequence $(c(t))_{t \geq 1 \text { squarefree }}$ where $t$ runs over positive squarefree integers only. Of course, similar questions can be studied for forms of weight $k+\frac{1}{2}$ in the plus subspace in which case $t$ has to be replaced by $|D|$ where $D$ is a fundamental discriminant with $(-1)^{k} D>0$. For a good (at least partial) survey the reader may look up the literature given in [4].

Note that sign change results trivially imply corresponding non-vanishing results and in general non-vanishing properties of Fourier coefficients a priori are easier to handle. We recall that non-vanishing of products of Fourier coefficients was studied in [3].

In this short note we will investigate sign change and non-vanishing properties of the double sequence $\left(c\left(4 n+r^{2}\right)\right)_{n \geq 1, r \in \mathbf{Z}}$ where $g$ is a cusp form of weight $k+\frac{1}{2}$ with $k$ even and level 4 in the plus subspace $S_{k+1 / 2}^{+}(\operatorname{so} c(m)=0$ unless $m \equiv 0,1(\bmod 4)$, see [7]). These

\section{Communicated by Jens Funke.}

Winfried Kohnen

winfried@mathi.uni-heidelberg.de

1 Mathematisches Institut der Universität, INF 205, 69120 Heidelberg, Germany 
coefficients turn up naturally when one considers the adjoint linear map with respect to the Petersson scalar products of (essentially) the linear map "multiplication with $\theta$ ", where

$$
\theta(z)=\sum_{r \in \mathbf{Z}} q^{r^{2}}
$$

is the standard theta function of weight $\frac{1}{2}$ and level 4 . Here as throughout $q=e^{2 \pi i z}$ for $z \in \mathcal{H}$, the complex upper half-plane.

Our results will be stated in the next section; the proofs will be given in section 3 . They rely on a detailed study of the above mentioned adjoint map, on growth properties of Fourier coefficients of cusp forms of integral weight due to Ram Murty and on a strong bound for the Fourier coefficients of cusp forms of half-integral weight due to Blomer-Harcos. Detailed references will be given below.

\section{Statement of results}

If $M \subset \mathbf{Z}$ we denote by $\# M$ the cardinality of $M$ (thus $\# M$ is either a non-negative integer or $\infty$ ).

By $k$ we always understand a positive even integer. We let $S_{k}$ be the space of cusp forms of weight $k$ on $\Gamma_{1}:=S L_{2}(\mathbf{Z})$. There is a linear map

$$
L: S_{k} \rightarrow S_{k+1 / 2}^{+}, \quad f(z) \mapsto f(4 z) \theta(z) .
$$

Note that in general $L$ is not Hecke equivariant.

We denote by $L^{*}: S_{k+1 / 2}^{+} \rightarrow S_{k}$ the linear map adjoint to $L$ with respect to the Petersson scalar products. Note that since $L$ is injective, $L^{*}$ is surjective.

Let $g \in S_{k+1 / 2}^{+}$be fixed, with Fourier coefficients $c(m)(m \geq 1)$. For each $n \in \mathbf{N}$ we then put

$$
\alpha_{n}:=\#\left\{r \in \mathbf{Z} \mid c\left(4 n+r^{2}\right) \neq 0\right\}
$$

and if in addition the $c(m)$ are real

$$
\alpha_{n}^{+}:=\#\left\{r \in \mathbf{Z} \mid c\left(4 n+r^{2}\right)>0\right\}, \quad \alpha_{n}^{-}:=\#\left\{r \in \mathbf{Z} \mid c\left(4 n+r^{2}\right)<0\right\} .
$$

Theorem 1 Let $g \in S_{k+1 / 2}^{+}$with real Fourier coefficients $c(m)(m \geq 1)$ and suppose that $L^{*} g$ is a normalized Hecke eigenform. Then there are sequences $\left(n_{\nu}\right)_{v \geq 1}$ and $\left(m_{\mu}\right)_{\mu \geq 1}$ in $\mathbf{N}$ such that for any $\sigma<\frac{1}{16}$ one has $\lim _{\nu \rightarrow \infty} \frac{\alpha_{n_{v}}^{+}}{n_{v}^{\sigma}}=\infty$ and $\lim _{\mu \rightarrow \infty} \frac{\alpha_{m_{\mu}}^{-}}{m_{\mu}^{\sigma}}=\infty$. In particular one has $\lim _{\nu \rightarrow \infty} \alpha_{n_{v}}^{+}=\infty$ and $\lim _{\mu \rightarrow \infty} \alpha_{m_{\mu}}^{-}=\infty$.

Remark It is easy to see that for any normalized Hecke eigenform $F \in S_{k}$ there exists $g \in S_{k+1 / 2}^{+}$with real Fourier coefficients such that $F=L^{*} g$.

If we drop the assumption that $L^{*} g$ is an eigenform, we still can get non-vanishing results for the Fourier coefficients. Let us put $V:=i m L$ and denote by $V^{\perp}$ the orthogonal complement of $V$ in $S_{k+1 / 2}^{+}$. 
Theorem 2 Let $g \in S_{k+1 / 2}^{+}$with real Fourier coefficients $c(m)(m \geq 1)$ and suppose that $g$ is not contained in $V^{\perp}$. Then there exists a sequence $\left(n_{v}\right)_{v \geq 1}$ in $\mathbf{N}$ such that for any $\sigma<\frac{1}{16}$ one has $\lim _{v \rightarrow \infty} \frac{\alpha_{n_{v}}}{n_{v}^{\sigma}}=\infty$. In particular one has $\lim _{v \rightarrow \infty} \alpha_{n_{v}}=\infty$.

Remark Applying the above result with $g$ replaced by $g-g_{0}$ where $g_{0} \in V^{\perp}$ has Fourier coefficients $c_{0}(m)$, we obtain a corresponding statement with " $c\left(4 n+r^{2}\right) \neq 0$ " replaced by " $c\left(4 n+r^{2}\right) \neq c_{0}\left(4 n+r^{2}\right)$ " in the definition of $\alpha_{n}$. A corresponding assertion mutatis mutandis (and in the case where the $c_{0}(m)$ are real) of course is valid also in the context of Theorem 1.

\section{Proof of results}

We start with briefly indicating the explicit construction of the map $L^{*}$ adjoint to $L$ following [9, sect. 5], and [8], mutatis mutandis.

Let $g \in S_{k+1 / 2}^{+}$. The $n$-th Fourier coefficient of $L^{*} g$ is given by

$$
a\left(L^{*} g, n\right)=\frac{(4 \pi n)^{k-1}}{(k-2) !}\left\langle L^{*} g, P_{k, n}\right\rangle
$$

by the usual Petersson formula, where $P_{k, n}$ denotes the $n$-th Poincare series in $S_{k}$.

By definition

$$
\begin{aligned}
\left\langle L^{*} g, P_{k, n}\right\rangle & =\left\langle g(z), P_{k, n}(4 z) \theta(z)\right\rangle \\
= & \int_{\mathcal{F}} G(z) \overline{P_{k, n}(4 z)} y^{k} d V
\end{aligned}
$$

where $z=x+i y, d V=\frac{d x d y}{y^{2}}$ is the invariant measure, $\mathcal{F}$ is a fundamental domain for $\Gamma_{0}(4) \subset \Gamma_{1}$ and $G(z):=\sqrt{y} g(z) \overline{\theta(z)}$ behaves like a modular form of weight $k$ under $\Gamma_{0}(4)$. Recall that $\Gamma_{0}(4)$ consists of those matrices in $\Gamma_{1}$ whose left lower component is divisible by 4 . The integral in the last line above can be computed by the usual unfolding argument.

Altogether one finds that

$$
a\left(L^{*} g, n\right)=C_{k} \cdot n^{k-1} \cdot \ell(g, n)
$$

where $C_{k}$ is a real positive constant depending only on $k$ and

$$
\ell(g, n):=\sum_{r \in \mathbf{Z}} \frac{c\left(4 n+r^{2}\right)}{\left(4 n+r^{2}\right)^{k-1 / 2}} .
$$

The convergence of the sum is clear by the usual Hecke estimate for the coefficients $c(m)$ (observe that we may assume that $k \geq 4$, otherwise $S_{k+1 / 2}^{+}=\{0\}$ ). This gives an explicit description of the map $L^{*}$.

Since the $P_{k, n}(n \geq 1)$ generate $S_{k}$, we also see that $V^{\perp}=k e r L^{*}$ consists of those $g$ with the property that $\ell(g, n)=0$ for all $n \geq 1$.

For the proof of our results we also need $\Omega$-results for the Fourier coefficients $a(n)(n \geq 1)$ of cusp forms $f \in S_{k}$. Recall that for arithmetic functions $v, w$ with $w(n)$ ultimately strictly positive, one defines 


$$
v(n)=\Omega(w(n))
$$

if

$$
\limsup _{n \rightarrow \infty} \frac{|v(n)|}{w(n)}>0
$$

and if in addition $v$ is real-valued

$$
v(n)=\Omega_{+}(w(n))
$$

if

$$
\limsup _{n \rightarrow \infty} \frac{v(n)}{w(n)}>0
$$

and

$$
v(n)=\Omega_{-}(w(n))
$$

if

$$
\liminf _{n \rightarrow \infty} \frac{v(n)}{w(n)}<0 .
$$

Now recall that for $f \neq 0$ it was proved in [11] that

$$
a(n)=\Omega\left(n^{(k-1) / 2} \exp \left(c \frac{\log n}{\log \log n}\right)\right),
$$

and if in addition $f$ is a normalized Hecke eigenform

$$
a(n)=\Omega_{ \pm}\left(n^{(k-1) / 2} \exp \left(c_{ \pm} \frac{\log n}{\log \log n}\right)\right),
$$

where $c, c_{ \pm}$are positive constants depending only on $f$.

We shall now prove the first assertion of Theorem 1 . We put $F:=L^{*} g$ and denote by $A(n)(n \geq 1)$ the Fourier coefficients of $F$. According to (4) (applied with $\Omega_{+}$) we can choose a sequence $\left(n_{v}\right)_{v \geq 1}$ in $\mathbf{N}$ such that

$$
A\left(n_{v}\right)>0
$$

for all $v$ and

$$
\lim _{v \rightarrow \infty} \frac{A\left(n_{v}\right)}{n_{v}^{(k-1) / 2}} \exp \left(-c_{+} \frac{\log n_{\nu}}{\log \log n_{v}}\right)>0 .
$$

We claim that

$$
\lim _{v \rightarrow \infty} \frac{\alpha_{n_{v}}^{+}}{n_{v}^{\sigma}}=\infty
$$

for any $\sigma<\frac{1}{16}$. 
Suppose that this is not true, for a given $\sigma$. Then we can find a sequence $n_{v_{1}}<n_{v_{2}}<\ldots$ and $K>0$ such that

$$
\frac{\alpha_{n_{v_{\mu}}}^{+}}{n_{v_{\mu}}^{\sigma}} \leq K,
$$

for all $\mu \geq 1$.

It follows from (1) and (2) that

$$
\begin{array}{r}
A\left(n_{v_{\mu}}\right)=C_{k} \cdot n_{v_{\mu}}^{k-1} \cdot\left(\sum_{r}^{+} \frac{c\left(4 n_{v_{\mu}}+r^{2}\right)}{\left(4 n_{v_{\mu}}+r^{2}\right)^{k-1 / 2}}+\sum_{r}^{-} \frac{c\left(4 n_{v_{\mu}}+r^{2}\right)}{\left(4 n_{v_{\mu}}+r^{2}\right)^{k-1 / 2}}\right) \\
\leq C_{k} \cdot n_{v_{\mu}}^{k-1} \cdot \sum_{r}^{+} \frac{c\left(4 n_{v_{\mu}}+r^{2}\right)}{\left(4 n_{v_{\mu}}+r^{2}\right)^{k-1 / 2}},
\end{array}
$$

where $r$ in $\sum_{r}^{+}$runs over those $r \in \mathbf{Z}$ with $c\left(4 n_{v_{\mu}}+r^{2}\right)>0$ and $r$ in $\sum_{r}^{-}$runs over those $r$ with $c\left(4 n_{v_{\mu}}+r^{2}\right) \leq 0$. Note that the sum $\sum_{r}^{+}$is non-empty by (1) and (5) and for each fixed $\mu$ is finite by (7).

By [1] the Fourier coefficients $c(m)$ of $g$ can be estimated by

$$
c(m) \ll_{g, \epsilon} m^{k / 2-\delta+\epsilon} \quad(\epsilon>0)
$$

where one can take $\delta=\frac{1}{16}$. This estimate is slightly better than the Weil bound with $\delta=0$. It is important to us that the bound (9) holds for all $m \geq 1$. Bounds better than the Weil bound for $m$ squarefree were obtained in $[2,5,10]$.

Inserting (9) into (8) we obtain

$$
\begin{gathered}
A\left(n_{v_{\mu}}\right) \ll_{g, \epsilon} n_{v_{\mu}}^{k-1} \cdot \sum_{r}^{+} \frac{1}{\left(4 n_{v_{\mu}}+r^{2}\right)^{k / 2-1 / 2+\delta-\epsilon}} \\
\ll_{g, \epsilon} n_{v_{\mu}}^{k-1} \cdot \frac{\alpha_{n_{v_{\mu}}}^{+}}{\left(4 n_{v_{\mu}}\right)^{k / 2-1 / 2+\delta-\epsilon}} \\
\ll_{g, \epsilon, K} n_{v_{\mu}}^{k / 2-1 / 2-\delta+\epsilon+\sigma}
\end{gathered}
$$

where in the last line we have used (7). Choosing $\epsilon=\delta-\sigma=\frac{1}{16}-\sigma$ we therefore find that

$$
A\left(n_{v_{\mu}}\right) \ll_{g, \epsilon, K} n_{v_{\mu}}^{(k-1) / 2} .
$$

Letting $\mu$ going to $\infty$ we obtain a contradiction to (6).

This proves the assertion of Theorem 1 regarding $\alpha_{n}^{+}$. To obtain the assertion with $\alpha_{n}^{-}$one proceeds in the same way, mutatis mutandis, using (4) with $\Omega_{-}$. Finally to prove Theorem 2 , one again proceeds in the same way, using (3). Note that the assumption that $g \notin V^{\perp}$ is used to guarantee that $L^{*} g \neq 0$.

Funding Open Access funding enabled and organized by Projekt DEAL.

Open Access This article is licensed under a Creative Commons Attribution 4.0 International License, which permits use, sharing, adaptation, distribution and reproduction in any medium or format, as long as you give appropriate credit to the original author(s) and the source, provide a link to the Creative Commons 
licence, and indicate if changes were made. The images or other third party material in this article are included in the article's Creative Commons licence, unless indicated otherwise in a credit line to the material. If material is not included in the article's Creative Commons licence and your intended use is not permitted by statutory regulation or exceeds the permitted use, you will need to obtain permission directly from the copyright holder. To view a copy of this licence, visit http://creativecommons.org/licenses/by/4.0/.

\section{References}

1. Blomer, V., Harcos, G.: Hybrid bounds for twisted $L$-functions. J. Reine Angew. Mathematik 621, 53-79 (2008)

2. Bykovskii, V.A.: A trace formula for the scalar product of Hecke series and its applications. J. Math. Sci. (New York) 89, 915-932 (1998)

3. Hofmann, E., Kohnen, W.: On products of Fourier coefficients of cusp forms. Forum Math. 29(1), 245-250 (2017)

4. Inam, I., Wiese, G.: Fast computation of half integral weight modular forms. Preprint (2020)

5. Iwaniec, H.: Fourier coefficients of modular forms of half integral weight. Invent. Math. 87, 385-401 (1987)

6. Knopp, M., Kohnen, W., Pribitkin, W.: On the signs of Fourier coefficients of cusp forms. Ramanujan J. 7(1-3), 269-277 (2003)

7. Kohnen, W.: Modular forms of half-integral weight on $\Gamma_{0}$ (4). Math. Ann. 248(3), 249-266 (1980)

8. Kohnen, W.: Cusp forms and special values of certain Dirichlet series. Math. Z. 207, 657-660 (1991)

9. Kohnen, W.: On squares of Hecke eigenforms (to appear in Pure Appl. Math. Quarterly)

10. Petrov, I., Young, M.P.: A generalized cubic moment and the Petersson formula for newforms (to appear in Math. Ann)

11. Ram Murty, M.: Oscillations of Fourier coefficients of modular forms. Math. Ann. 262, 431-446 (1983)

Publisher's Note Springer Nature remains neutral with regard to jurisdictional claims in published maps and institutional affiliations. 